preprint IC/2002/115

\title{
Bhabha vs. Møller scattering as a contact-interaction analyzer at a polarized Linear Collider
}

\author{
A. Pankov ${ }^{a, b}$ and N. Paver ${ }^{a}$ \\ ${ }^{a}$ Dipartimento di Fisica Teorica, Università di Trieste and \\ Istituto Nazionale di Fisica Nucleare, Sezione di Trieste, Trieste, Italy \\ ${ }^{b}$ The Abdus Salam International Centre for Theoretical Physics, Trieste, Italy
}

\begin{abstract}
We discuss electron-electron contact-interaction searches in the processes $e^{+} e^{-} \rightarrow e^{+} e^{-}$ and $e^{-} e^{-} \rightarrow e^{-} e^{-}$at planned Linear Colliders run in the $e^{+} e^{-}$and $e^{-} e^{-}$modes with both beams longitudinally polarized. Our analysis is based on the measurement, for the two processes, of polarized differential cross sections, and allows to simultaneously take into account the general set of electron contact interaction couplings as independent, non-zero, parameters thus avoiding the simplifying choice of a model. We evaluate the corresponding model-independent constraints on the contact coupling constants, emphasizing the role of the available beam polarization and the complementarity, as far as the chirality of the constants is concerned, of the two processes in giving the best constraints. We also make a comparison with the potential of $e^{+} e^{-} \rightarrow \mu^{+} \mu^{-}$at the same energy and initial beams polarization.
\end{abstract}




\section{Introduction}

Contact interaction Lagrangians (CI) provide a framework to account for the phenomenological effects of non-standard dynamics characterized by extremely large intrinsic mass scales $\Lambda$, at the 'low' energies $\sqrt{s} \ll \Lambda$ attainable at current particle accelerators. One of the historical motivations for considering such a framework is the fact that 'low energy' manifestations of quark and lepton substructure would occur via four-fermion quark and lepton contact interactions, induced by exchanges of quite heavy sub-constituent bound states with mass of the order of $\Lambda$. Indeed, in the spirit of the 'effective interactions', this concept can be used more generally, to parameterize non-standard, very heavy particle exchanges in reactions among quarks and leptons, in the form of 'low energy' expansions of the relevant amplitudes at the leading order in $\sqrt{s} / \Lambda$. Since the above mentioned exchanged heavy particles, with mass $M \gg M_{W, Z}$, could not be directly produced at the collider energy $\sqrt{s}$, the underlying non-standard dynamics could experimentally manifest itself only indirectly, by deviations of the measured observables from the Standard Model (SM) predictions. If such deviations were effectively observed to a given significance level, one could try to gain numerical information on the parameters (masses and coupling constants) of non-standard models and, eventually, select the viable ones [1, 2]. In the case where, instead, no deviation from the SM predictions is observed within the experimental accuracy, one can set numerical bounds or constraints on the parameters characterizing the new interactions, and determine the discovery reach of planned high-energy colliders. Clearly, also this kind of information should be phenomenologically useful in model applications.

The explicit form of the contact interaction Lagrangian depends on the kind of external particles participating in the considered reaction. For the Bhabha scattering process of interest here:

$$
e^{+}+e^{-} \rightarrow e^{+}+e^{-}
$$

as well as for Møller scattering:

$$
e^{-}+e^{-} \rightarrow e^{-}+e^{-}
$$

we consider the four-fermion contact-interaction Lagrangian [3]:

$$
\mathcal{L}_{\mathrm{CI}}=\frac{1}{1+\delta_{e f}} \sum_{i, j} g_{\mathrm{eff}}^{2} \epsilon_{i j}\left(\bar{e}_{i} \gamma_{\mu} e_{i}\right)\left(\bar{f}_{j} \gamma^{\mu} f_{j}\right) .
$$

In Eq. (3) : $i, j=\mathrm{L}, \mathrm{R}$ denote left- or right-handed fermion helicities, $\delta_{e f}=1$ for processes (11) and (2) and, if we assumed lepton universality, the same Lagrangian, with $\delta_{e f}=0$, is relevant to the annihilation processes

$$
e^{+}+e^{-} \rightarrow l^{+}+l^{-}
$$

with $l=\mu, \tau$. The CI coupling constants in Eq. (3) are parameterized in terms of corresponding mass scales as $\epsilon_{i j}=\eta_{i j} / \Lambda_{i j}^{2}$ and, according to the previous remarks concerning compositeness, one assumes $g_{\text {eff }}^{2}=4 \pi$. Also, by convention, one takes $\left|\eta_{i j}\right|=1$ or 
$\eta_{i j}=0$, leaving the energy scales $\Lambda_{i j}$ as free, a priori independent, parameters. The explicit $S U(3) \times S U(2) \times U(1)$ symmetry of the helicity conserving four-fermion lepton contact interaction (3) reflects that the new dynamics are active well-beyond the electroweak scale. Furthermore, Eq. (3) represents the lowest dimensional operator, $D=6$ being the minimum, and higher-dimensional operators, suppressed by higher powers of $s / \Lambda^{2}$, are supposed to be negligible.

As anticipated, we will study the effects of the interaction (3i) in processes (11) and (21) at an $e^{+} e^{-}$Linear Collider with c.m. energy $\sqrt{s}=0.5 \mathrm{TeV}$ and polarized electron and positron beams [4. Indeed, the possibility of studying $e^{-} e^{-}$initiated processes, in particular new physics, at such a facility by turning the positron beam into an electron one, has been recently considered with interest [5]. Therefore, it should be useful to evaluate, and compare, the sensitivities to the CI coupling constants that can be obtained from the measurements of processes (11) and (2).

Clearly, from current lower bounds on $\Lambda$ 's obtained at LEP [6, 7], of the order of 10-15 $\mathrm{TeV}$ depending on the specific models chosen to fit the data, we can assume $s \ll \Lambda^{2}$, so that the relative size of the deviations from the SM induced by Eq. (3) is expected to be of order $s / \alpha \Lambda^{2}$, with $\alpha$ the SM coupling (essentially, the fine structure constant), and therefore to be quite small 1 Consequently, very high collider energies and luminosities are required to attain a significant sensitivity on these effects.

We notice that for the case of the Bhabha process (11), Eq. (3) envisages the existence of six independent CI models, each one contributing to individual helicity amplitudes or combinations of them, with a priori free, and nonvanishing, coefficients (basically, $\epsilon_{\mathrm{LL}}, \epsilon_{\mathrm{RR}}$ and $\epsilon_{\mathrm{LR}}=\epsilon_{\mathrm{RL}}$ combined with the \pm signs). The same is true for the Møller process (2) 2 Correspondingly, in principle, a model-independent analysis of the data should account for the situation where the full Eq. (3) is included in the expression for the cross section. Potentially, in this case, the different CI couplings may interfere and such interference could substantially weaken the bounds because, although the different helicity amplitudes by themselves do not interfere, the deviations from the SM could be positive for one helicity amplitude and negative for another, so that accidental cancellations might occur in the sought for deviations of the relevant observables from the SM predictions.

The analysis of processes (11) and (2) proposed here relies on the initial beams longitudinal polarization envisaged at the planned Linear Colliders. The polarization can be exploited to extract the values of the individual helicity cross sections from suitable combinations of measurable polarized cross sections and, consequently, to disentangle the effects of the corresponding CI constants $\epsilon_{i j}$, see, e.g., Ref. [10]. Therefore, all CI couplings of Eq. (3) are simultaneously included as independent, non vanishing, free parameters and, yet, separate constraints (or exclusion regions) on their values can be obtained, free from potential weakening due to accidental cancellations. In this sense, the procedure should be considered as model-independent. We will also make a comparison of the results with those

\footnotetext{
${ }^{1}$ For bounds from different kinds of processes, in particular on contact couplings to quarks, see, e.g., Refs. [8, 9 .

${ }^{2}$ In general, apart from the \pm possibility, for $e^{+} e^{-} \rightarrow \bar{f} f$ with $f \neq e$ there are four independent CI couplings, so that in the present case of processes (11) and (2) there is one free parameter less.
} 
obtained from the simplest, model-dependent, procedure of assuming non-zero values for only one of the couplings (or one specific combination of them) at a time, with all others set to zero.

Specifically, in Sect. 2 we introduce the polarized observables for the Bhabha and the Møller processes, Eqs. (11) and (2), and discuss the sensitivities of the different angular ranges to the CI couplings in the two cases. In Sect. 3 we perform the numerical analysis, based on a $\chi^{2}$ procedure, to derive the constraints on the CI couplings and establish the attainable reach on the mass scales $\Lambda_{i j}$ as a function of the integrated luminosity. Sect. 4 contains some conclusive remarks, in particular a comparison of the results from the two processes, and with those obtained from the annihilation process (4).

\section{Polarized observables}

\subsection{Bhabha scattering}

With $P^{-}$and $P^{+}$the longitudinal polarization of the electron and positron beams, respectively, and $\theta$ the angle between the incoming and the outgoing electrons in the c.m. frame, the differential cross section of process (11) at lowest order, including $\gamma$ and $Z$ exchanges

both in the $s$ and $t$ channels and the contact interaction (13), can be written in the following form [11-15]:

$$
\begin{aligned}
\frac{\mathrm{d} \sigma\left(P^{-}, P^{+}\right)}{\mathrm{d} \cos \theta} & =\frac{\left(1+P^{-}\right)\left(1-P^{+}\right)}{4} \frac{\mathrm{d} \sigma_{\mathrm{R}}}{\mathrm{d} \cos \theta}+\frac{\left(1-P^{-}\right)\left(1+P^{+}\right)}{4} \frac{\mathrm{d} \sigma_{\mathrm{L}}}{\mathrm{d} \cos \theta} \\
& +\frac{1+P^{-} P^{+}}{2} \frac{\mathrm{d} \sigma_{\mathrm{LR}, t}}{\mathrm{~d} \cos \theta} .
\end{aligned}
$$

In Eq. (5) :

$$
\begin{aligned}
\frac{\mathrm{d} \sigma_{\mathrm{L}}}{\mathrm{d} \cos \theta} & =\frac{\mathrm{d} \sigma_{\mathrm{LL}}}{\mathrm{d} \cos \theta}+\frac{\mathrm{d} \sigma_{\mathrm{LR}, s}}{\mathrm{~d} \cos \theta}, \\
\frac{\mathrm{d} \sigma_{\mathrm{R}}}{\mathrm{d} \cos \theta} & =\frac{\mathrm{d} \sigma_{\mathrm{RR}}}{\mathrm{d} \cos \theta}+\frac{\mathrm{d} \sigma_{\mathrm{RL}, s}}{\mathrm{~d} \cos \theta},
\end{aligned}
$$

with

$$
\begin{array}{ll}
\frac{\mathrm{d} \sigma_{\mathrm{LL}}}{\mathrm{d} \cos \theta}=\frac{2 \pi \alpha^{2}}{s}\left|A_{\mathrm{LL}}\right|^{2}, & \frac{\mathrm{d} \sigma_{\mathrm{RR}}}{\mathrm{d} \cos \theta}=\frac{2 \pi \alpha^{2}}{s}\left|A_{\mathrm{RR}}\right|^{2}, \\
\frac{\mathrm{d} \sigma_{\mathrm{LR}, t}}{\mathrm{~d} \cos \theta}=\frac{2 \pi \alpha^{2}}{s}\left|A_{\mathrm{LR}, t}\right|^{2}, & \frac{\mathrm{d} \sigma_{\mathrm{LR}, s}}{\mathrm{~d} \cos \theta}=\frac{\mathrm{d} \sigma_{\mathrm{RL}, s}}{\mathrm{~d} \cos \theta}=\frac{2 \pi \alpha^{2}}{s}\left|A_{\mathrm{LR}, s}\right|^{2},
\end{array}
$$

and

$$
\begin{aligned}
& A_{\mathrm{RR}}=\frac{u}{s}\left[1+\frac{s}{t}+g_{\mathrm{R}}^{2}\left(\chi_{Z}(s)+\frac{s}{t} \chi_{Z}(t)\right)+2 \frac{s}{\alpha} \epsilon_{\mathrm{RR}}\right] \\
& A_{\mathrm{LL}}=\frac{u}{s}\left[1+\frac{s}{t}+g_{\mathrm{L}}^{2}\left(\chi_{Z}(s)+\frac{s}{t} \chi_{Z}(t)\right)+2 \frac{s}{\alpha} \epsilon_{\mathrm{LL}}\right]
\end{aligned}
$$




$$
\begin{aligned}
& A_{\mathrm{LR}, s}=\frac{t}{s}\left[1+g_{\mathrm{R}} g_{\mathrm{L}} \chi_{Z}(s)+\frac{s}{\alpha} \epsilon_{\mathrm{LR}}\right], \\
& A_{\mathrm{LR}, t}=\frac{s}{t}\left[1+g_{\mathrm{R}} g_{\mathrm{L}} \chi_{Z}(t)+\frac{t}{\alpha} \epsilon_{\mathrm{LR}}\right] .
\end{aligned}
$$

Here: $\alpha$ is the fine structure constant; $t=-s(1-\cos \theta) / 2, u=-s(1+\cos \theta) / 2$ and $\chi_{Z}(s)=s /\left(s-M_{Z}^{2}+i M_{Z} \Gamma_{Z}\right)$ and $\chi_{Z}(t)=t /\left(t-M_{Z}^{2}\right)$ represent the $Z$ propagator in the $s$ and $t$ channels, respectively, with $M_{Z}$ and $\Gamma_{Z}$ the mass and width of the $Z ; g_{\mathrm{R}}=\tan \theta_{W}$, $g_{\mathrm{L}}=-\cot 2 \theta_{W}$ are the SM right- and left-handed electron couplings of the $Z$, with $\theta_{W}$ the electroweak mixing angle.

With both beams polarized, the polarization of each beam can be changed on a pulse by pulse basis. This would allow the separate measurement of the polarized cross sections for each of the three polarization configurations,+++- and -+ , corresponding to the sets of beam polarizations $\left(P^{-}, P^{+}\right)=\left(P_{1}, P_{2}\right),\left(P_{1},-P_{2}\right)$ and $\left(-P_{1}, P_{2}\right)$, respectively, with $P_{1,2}>0$. Specifically, from Eq. (5), with the simplifying notation $\mathrm{d} \sigma \equiv \mathrm{d} \sigma / \mathrm{d} \cos \theta$ :

$$
\begin{aligned}
& \mathrm{d} \sigma_{++}=\frac{\left(1+P_{1}\right)\left(1-P_{2}\right)}{4} \mathrm{~d} \sigma_{\mathrm{R}}+\frac{\left(1-P_{1}\right)\left(1+P_{2}\right)}{4} \mathrm{~d} \sigma_{\mathrm{L}}+\frac{1+P_{1} P_{2}}{2} \mathrm{~d} \sigma_{\mathrm{LR}, t}, \\
& \mathrm{~d} \sigma_{+-}=\frac{\left(1+P_{1}\right)\left(1+P_{2}\right)}{4} \mathrm{~d} \sigma_{\mathrm{R}}+\frac{\left(1-P_{1}\right)\left(1-P_{2}\right)}{4} \mathrm{~d} \sigma_{\mathrm{L}}+\frac{1-P_{1} P_{2}}{2} \mathrm{~d} \sigma_{\mathrm{LR}, t}, \\
& \mathrm{~d} \sigma_{-+}=\frac{\left(1-P_{1}\right)\left(1-P_{2}\right)}{4} \mathrm{~d} \sigma_{\mathrm{R}}+\frac{\left(1+P_{1}\right)\left(1+P_{2}\right)}{4} \mathrm{~d} \sigma_{\mathrm{L}}+\frac{1-P_{1} P_{2}}{2} \mathrm{~d} \sigma_{\mathrm{LR}, t} .
\end{aligned}
$$

To extract from the measured polarized cross sections the values of $\mathrm{d} \sigma_{\mathrm{R}}, \mathrm{d} \sigma_{\mathrm{L}}$ and $\mathrm{d} \sigma_{\mathrm{LR}, t}$, that carry the information on the CI couplings, one has to invert the system of equations (91). The solution reads:

$$
\begin{aligned}
\mathrm{d} \sigma_{\mathrm{R}} & =\frac{\left(1+P_{2}\right)^{2}}{2 P_{2}\left(P_{1}+P_{2}\right)} \mathrm{d} \sigma_{+-}+\frac{\left(1-P_{1}\right)^{2}}{2 P_{1}\left(P_{1}+P_{2}\right)} \mathrm{d} \sigma_{-+}-\frac{1-P_{1} P_{2}}{2 P_{1} P_{2}} \mathrm{~d} \sigma_{++}, \\
\mathrm{d} \sigma_{\mathrm{L}} & =\frac{\left(1-P_{2}\right)^{2}}{2 P_{2}\left(P_{1}+P_{2}\right)} \mathrm{d} \sigma_{+-}+\frac{\left(1+P_{1}\right)^{2}}{2 P_{1}\left(P_{1}+P_{2}\right)} \mathrm{d} \sigma_{-+}-\frac{1-P_{1} P_{2}}{2 P_{1} P_{2}} \mathrm{~d} \sigma_{++}, \\
\mathrm{d} \sigma_{\mathrm{LR}, t} & =-\frac{1-P_{2}^{2}}{2 P_{2}\left(P_{1}+P_{2}\right)} \mathrm{d} \sigma_{+-}-\frac{1-P_{1}^{2}}{2 P_{1}\left(P_{1}+P_{2}\right)} \mathrm{d} \sigma_{-+}+\frac{1+P_{1} P_{2}}{2 P_{1} P_{2}} \mathrm{~d} \sigma_{++} .
\end{aligned}
$$

As one can see from Eqs.([6)-(8),$\sigma_{\mathrm{LR}, t}$ depends on a single contact interaction parameter $\left(\epsilon_{\mathrm{LR}}\right)$, while $\sigma_{\mathrm{R}}$ and $\sigma_{\mathrm{L}}$ depend on pairs of parameters, $\left(\epsilon_{\mathrm{RR}}, \epsilon_{\mathrm{LR}}\right)$ and $\left(\epsilon_{\mathrm{LL}}, \epsilon_{\mathrm{LR}}\right)$, respectively. Therefore, the derivation of the model-independent constraints on the CI couplings requires the combination of all polarized cross sections as in Eq. (10). In this regard, to emphasize the role of polarization, one can easily notice from Eqs. (15)-(8) that in the unpolarized case $P_{1}=P_{2}=0$, the interference of the $\epsilon_{\mathrm{LR}}$ term with the SM amplitude in $A_{\mathrm{LR} s}$ and $A_{\mathrm{LR}, t}$ has opposite signs, leading to a partial cancellation for $-t \sim s$. Consequently, as briefly anticipated in Sect. 1, one can expect the unpolarized cross section to have reduced sensitivity to $\epsilon_{\mathrm{LR}}$. Conversely, $\epsilon_{\mathrm{LR}}$ is directly accessible from $\mathrm{d} \sigma_{\mathrm{LR}, t}$, via polarized cross sections as in Eq. (10). Also, considering that numerically $g_{\mathrm{L}}^{2} \cong g_{\mathrm{R}}^{2}$, the parameters $\epsilon_{\mathrm{LL}}$ 
and $\epsilon_{\mathrm{RR}}$ contribute to the unpolarized cross section through $A_{\mathrm{RR}}$ and $A_{\mathrm{LL}}$ with equal coefficients, so that, in general, only correlations of the form $\left|\epsilon_{\mathrm{LL}}+\epsilon_{\mathrm{RR}}\right|<\mathrm{const}$, and not finite allowed regions, could be derived in the unpolarized case.

To make contact to the experimental situation we take $P_{1}=0.8$ and $P_{2}=0.6$, and impose a cut in the forward and backward directions. Specifically, we consider the cut angular range $|\cos \theta|<0.9$ and divide it into nine equal-size bins of width $\Delta z=0.2$ $(z \equiv \cos \theta)$. We also introduce the experimental efficiency, $\epsilon$, for detecting the final $e^{+} e^{-}$ pair and $\epsilon \simeq 100 \%$ is assumed.

We then define the three, directly measurable, event rates integrated over each bin:

$$
N_{++}, \quad N_{+-}, \quad N_{-+},
$$

and $(\alpha \beta=++$, etc. $)$ :

$$
N_{\alpha \beta}^{\text {bin }}=\frac{1}{3} \mathcal{L}_{\text {int }}\left(e^{+} e^{-}\right) \epsilon \int_{\text {bin }}\left(\mathrm{d} \sigma_{\alpha \beta} / \mathrm{d} z\right) \mathrm{d} z .
$$

In Eq. (12), $\mathcal{L}_{\text {int }}$ is the time-integrated luminosity, which is assumed to be equally divided among the three combinations of electron and positron beam polarizations defined in Eqs. (91).

In Fig. 1, the bin-integrated angular distributions of $N_{++}^{\text {bin }}$ and $N_{+-}^{\text {bin }}$ in the SM at

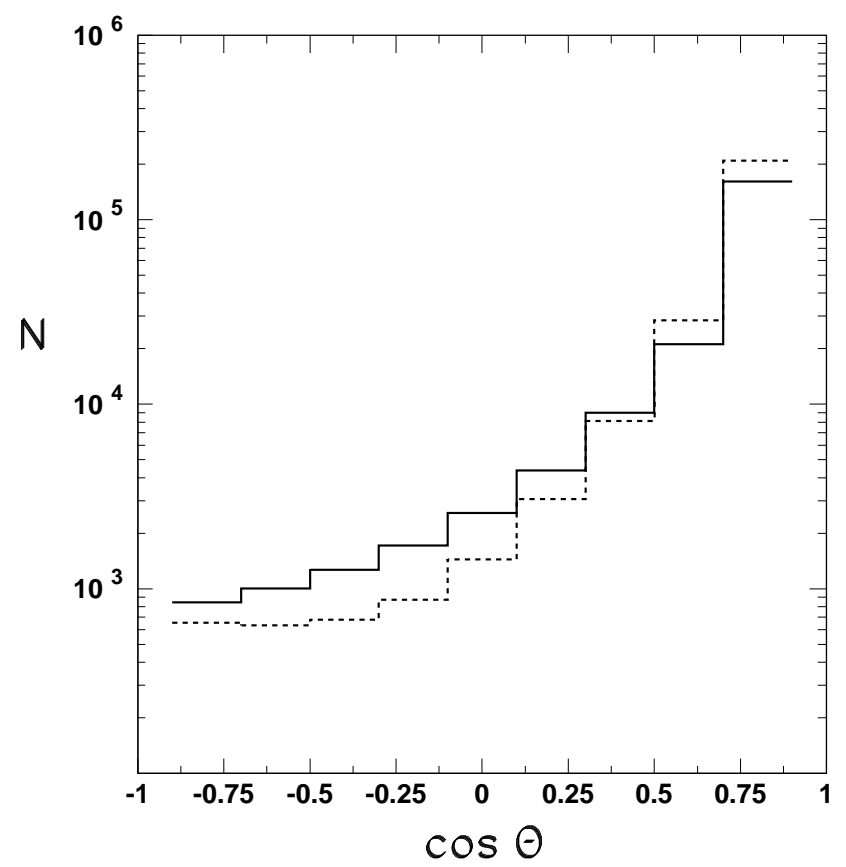

Figure 1: Bin-integrated angular distributions of $N_{++}^{\text {bin }}$ (solid line) and $N_{+-}^{\text {bin }}$ (dashed line), Eq.(12), in the SM at $\sqrt{s}=500 \mathrm{GeV}, \mathcal{L}_{\text {int }}\left(e^{+} e^{-}\right)=50 \mathrm{fb}^{-1},\left|P^{-}\right|=0.8$ and $\left|P^{+}\right|=0.6$.

$\sqrt{s}=500 \mathrm{GeV}$ and $\mathcal{L}_{\text {int }}=50 \mathrm{fb}^{-1}$ are presented as histograms. Here, the SM cross 
sections have been evaluated by means of the effective Born approximation [16, 17]. The typical forward peak, dominated by the $t$-channel photon pole, dramatically shows up, and determines a really large statistics available in the region of small $t$. The $\cos \theta$ distribution for the remaining polarization configuration $N_{-+}^{\text {bin }}$ in (9) is similar and, therefore, we do not represent it here.

The next step is to define the relative deviations of the polarized cross sections from the SM predictions, due to the contact interaction. In general, for such deviations, we use the notation:

$$
\Delta(\mathcal{O})=\frac{\mathcal{O}(S M+C I)-\mathcal{O}(S M)}{\mathcal{O}(S M)}
$$

where $\mathcal{O}=\sigma_{\mathrm{R}}, \sigma_{\mathrm{L}}$ and $\sigma_{\mathrm{LR}, t}$. To get an illustration of the effect of the contact interactions on the observables (10) under consideration, we show in Figs. 2a,b,c the angular distributions of the relative deviations of $\mathrm{d} \sigma_{\mathrm{R}}$ and $\mathrm{d} \sigma_{\mathrm{LR}, t}$, taking as examples the values of $\mathcal{L}_{\text {int }}$ and $\Lambda_{i j}$ indicated in the caption. The SM predictions are evaluated in the same effective Born approximation as in Fig. 1. The deviations $\Delta(\mathcal{O})$ are then compared to the expected statistical relative uncertainties, represented by the vertical bars. Figs. 2a,c show that d $\sigma_{\mathrm{R}}$ is sensitive to the contact interaction $\epsilon_{\mathrm{RR}}$ in the forward region, where the ratio of the "signal' to the statistical uncertainty substantially increases, while it is sensitive to $\epsilon_{\mathrm{LR}}$ in the backward direction. Also, it qualitatively indicates that, for the chosen values of the c.m. energy $\sqrt{s}$ and $\mathcal{L}_{\text {int }}$, the reach on $\Lambda_{\mathrm{RR}}$ will be substantially larger than $30 \mathrm{TeV}$. Conversely, Fig. $2 \mathrm{~b}$ shows that the sensitivity of $\mathrm{d} \sigma_{\mathrm{LR}, t}$ is almost independent on the chosen kinemat-
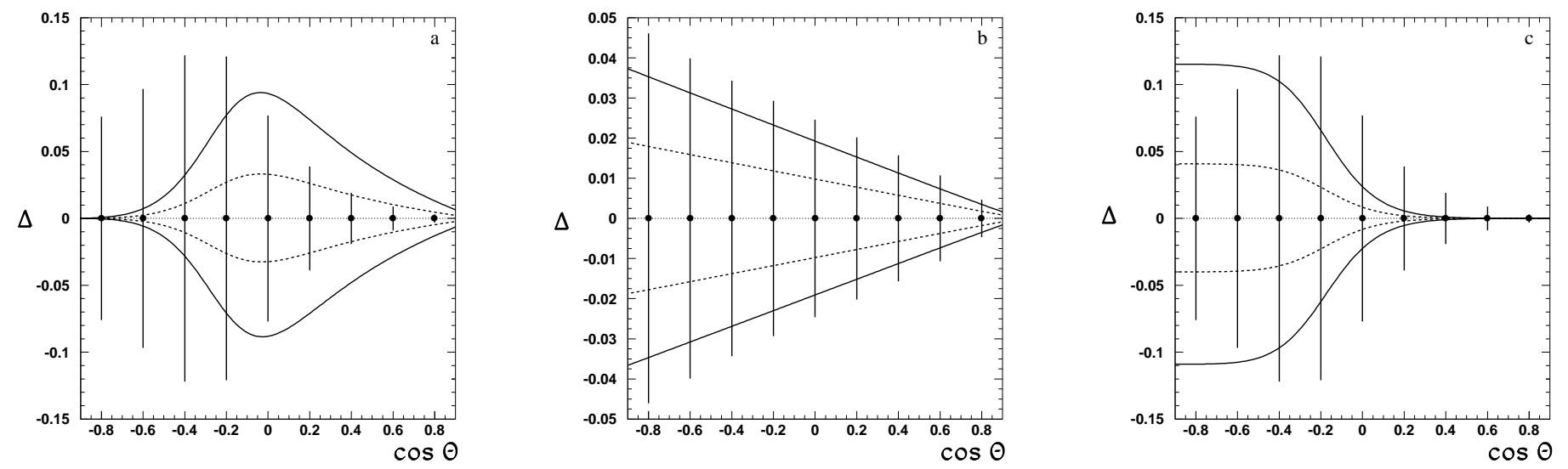

Figure 2: The angular distributions of relative deviations from the SM predictions: (a) $\Delta\left(\sigma_{\mathrm{R}}\right)$ for $\Lambda_{\mathrm{RR}}=30 \mathrm{TeV}$ (solid line) and $50 \mathrm{TeV}$ (dashed line); (b) $\Delta\left(\sigma_{\mathrm{LR}, t}\right)$ for $\Lambda_{\mathrm{LR}}=50$ $\mathrm{TeV}$ (solid line) and $70 \mathrm{TeV}$ (dashed line); (c) $\Delta\left(\sigma_{\mathrm{R}}\right)$ for $\Lambda_{\mathrm{LR}}=30 \mathrm{TeV}$ (solid line) and 50 $\mathrm{TeV}$ (dashed line). In (a) and (b) the curves above (below) the horizontal line correspond to negative (positive) interference between contact interaction and SM amplitude, whereas the opposite occurs in (c). The error bars show the expected statistical error at $\mathcal{L}_{\text {int }}\left(e^{+} e^{-}\right)=$ $50 \mathrm{fb}^{-1}$. 

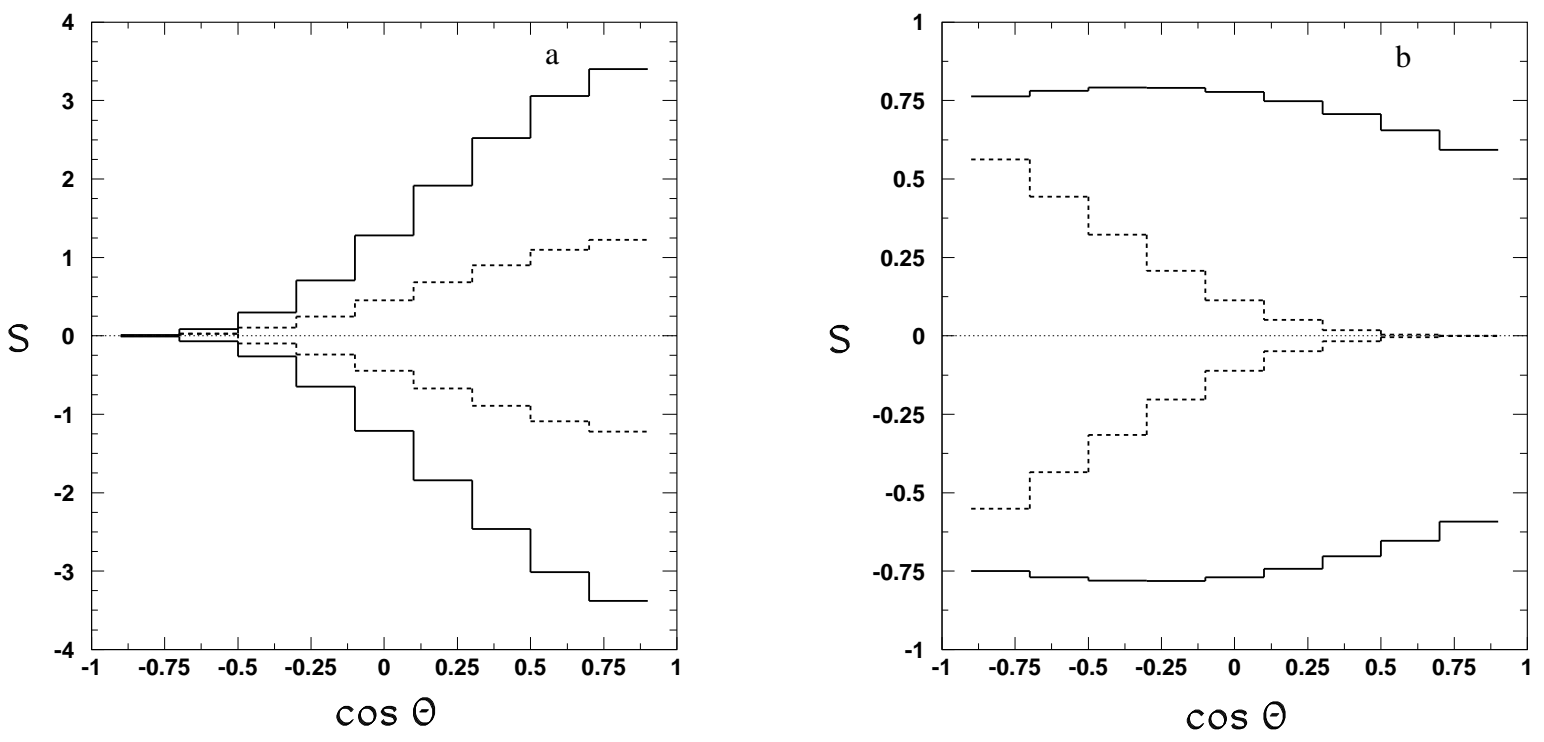

Figure 3: (a) Statistical significance $\mathcal{S}\left(\sigma_{R}\right)$ as a function of $\cos \theta$ at $\Lambda_{\mathrm{RR}}=30 \mathrm{TeV}$ (solid line) and $50 \mathrm{TeV}$ (dashed line); (b) Statistical significance $\mathcal{S}\left(\sigma_{R}\right)$ (dashed line) and $\mathcal{S}\left(\sigma_{L R, t}\right)$ (solid line) as a function of $\cos \theta$ at $\Lambda_{\mathrm{LR}}=50 \mathrm{TeV}$. Here: $\sqrt{s}=500 \mathrm{GeV}, \mathcal{L}_{\text {int }}\left(e^{+} e^{-}\right)=$ $50 \mathrm{fb}^{-1},\left|P^{-}\right|=0.8$ and $\left|P^{+}\right|=0.6$

ical range in $\cos \theta$, leading to a really high sensitivity of this observable to $\epsilon_{\mathrm{LR}}$, and to a corresponding reach on $\Lambda_{\mathrm{LR}}$ potentially larger than $50 \mathrm{TeV}$. The corresponding behaviour of the statistical significances, defined as the ratio between the deviation from the SM and the statistical uncertainty for each bin, $\mathcal{S}(\mathcal{O})=\Delta(\mathcal{O}) / \delta \mathcal{O}$ with $\delta \mathcal{O}$ the expected statistical relative uncertainty, are shown in Figs. $3 \mathrm{a}, \mathrm{b}$

\subsection{Møller scattering}

With $P_{1}^{-}$and $P_{2}^{-}$the longitudinal polarization of the electron beams, the differential cross section of process (2) can be written in the following form [18 20, 4]

$$
\begin{aligned}
\frac{\mathrm{d} \sigma\left(P_{1}^{-}, P_{2}^{-}\right)}{\mathrm{d} \cos \theta} & =\frac{\left(1+P_{1}^{-}\right)\left(1+P_{2}^{-}\right)}{4} \frac{\mathrm{d} \sigma_{\mathrm{RR}}}{\mathrm{d} \cos \theta}+\frac{\left(1-P_{1}^{-}\right)\left(1-P_{2}^{-}\right)}{4} \frac{\mathrm{d} \sigma_{\mathrm{LL}}}{\mathrm{d} \cos \theta} \\
& +\frac{1-P_{1}^{-} P_{2}^{-}}{2}\left(\frac{\mathrm{d} \sigma_{\mathrm{LR}, t}}{\mathrm{~d} \cos \theta}+\frac{\mathrm{d} \sigma_{L R, u}}{\mathrm{~d} \cos \theta}\right) .
\end{aligned}
$$

\footnotetext{
${ }^{3}$ One can notice from Eq. (8) that the statistical significance $\mathcal{S}$ goes to zero in the limit $\theta \rightarrow 0$. This is not evident from Figs. 3a,b due to the limited kinematical region $|\cos \theta|<0.9$ taken in our analysis.

${ }^{4}$ In the case of Møller scattering one can find for the cross section results similar to Bhabha scattering, that can be obtained by crossing symmetry except for the overall normalization factor $1 / 2$ related to identical particles.
} 
In Eq. (14):

$$
\begin{array}{ll}
\frac{\mathrm{d} \sigma_{\mathrm{RR}}}{\mathrm{d} \cos \theta}=\frac{\pi \alpha^{2}}{s}\left|A_{\mathrm{RR}}\right|^{2}, & \frac{\mathrm{d} \sigma_{\mathrm{LL}}}{\mathrm{d} \cos \theta}=\frac{\pi \alpha^{2}}{s}\left|A_{\mathrm{LL}}\right|^{2} \\
\frac{\mathrm{d} \sigma_{\mathrm{LR}, u}}{\mathrm{~d} \cos \theta}=\frac{\pi \alpha^{2}}{s}\left|A_{\mathrm{LR}, u}\right|^{2}, & \frac{\mathrm{d} \sigma_{\mathrm{LR}, t}}{\mathrm{~d} \cos \theta}=\frac{\pi \alpha^{2}}{s}\left|A_{\mathrm{LR}, t}\right|^{2}
\end{array}
$$

and

$$
\begin{aligned}
A_{\mathrm{RR}} & =\frac{s}{t}\left[1+\frac{t}{u}+g_{\mathrm{R}}^{2}\left(\chi_{Z}(t)+\frac{t}{u} \chi_{Z}(u)\right)+2 \frac{t}{\alpha} \epsilon_{\mathrm{RR}}\right] \\
A_{\mathrm{LL}} & =\frac{s}{t}\left[1+\frac{t}{u}+g_{\mathrm{L}}^{2}\left(\chi_{Z}(t)+\frac{t}{u} \chi_{Z}(u)\right)+2 \frac{t}{\alpha} \epsilon_{\mathrm{LL}}\right] \\
A_{\mathrm{LR}, u} & =\frac{t}{u}\left[1+g_{\mathrm{R}} g_{\mathrm{L}} \chi_{Z}(u)+\frac{u}{\alpha} \epsilon_{\mathrm{LR}}\right] \\
A_{\mathrm{LR}, t} & =\frac{u}{t}\left[1+g_{\mathrm{R}} g_{\mathrm{L}} \chi_{Z}(t)+\frac{t}{\alpha} \epsilon_{\mathrm{LR}}\right],
\end{aligned}
$$

where $\chi_{Z}(u)=u /\left(u-M_{Z}^{2}\right)$. Notice that the amplitudes $A_{i j}$ are now functions of $t$ and $u$ instead of $t$ and $s$ as in the case of Bhabha scattering.

As for the previous process, with both beams polarized the polarization of each electron beam can be changed on a pulse by pulse basis. This would allow the separate measurement of the polarized cross sections for each of the three polarization configurations,++-and +- , corresponding to the sets of beam polarizations $\left(P_{1}^{-}, P_{2}^{-}\right)=\left(P_{1}, P_{2}\right),\left(-P_{1},-P_{2}\right)$ and $\left(P_{1},-P_{2}\right)$, respectively, with $P_{1,2}>0$. From Eq. (14):

$$
\begin{aligned}
& \mathrm{d} \sigma_{++}=\frac{\left(1+P_{1}\right)\left(1+P_{2}\right)}{4} \mathrm{~d} \sigma_{\mathrm{RR}}+\frac{\left(1-P_{1}\right)\left(1-P_{2}\right)}{4} \mathrm{~d} \sigma_{\mathrm{LL}}+\frac{1-P_{1} P_{2}}{2} \mathrm{~d} \sigma_{\mathrm{LR}}, \\
& \mathrm{d} \sigma_{--}=\frac{\left(1-P_{1}\right)\left(1-P_{2}\right)}{4} \mathrm{~d} \sigma_{\mathrm{RR}}+\frac{\left(1+P_{1}\right)\left(1+P_{2}\right)}{4} \mathrm{~d} \sigma_{\mathrm{LL}}+\frac{1-P_{1} P_{2}}{2} \mathrm{~d} \sigma_{\mathrm{LR}}, \\
& \mathrm{d} \sigma_{+-}=\frac{\left(1+P_{1}\right)\left(1-P_{2}\right)}{4} \mathrm{~d} \sigma_{\mathrm{RR}}+\frac{\left(1-P_{1}\right)\left(1+P_{2}\right)}{4} \mathrm{~d} \sigma_{\mathrm{LL}}+\frac{1+P_{1} P_{2}}{2} \mathrm{~d} \sigma_{\mathrm{LR}} .
\end{aligned}
$$

To extract from the measured polarized cross sections the values of $\mathrm{d} \sigma_{\mathrm{RR}}, \mathrm{d} \sigma_{\mathrm{LL}}$ and $\mathrm{d} \sigma_{\mathrm{LR}}$, that carry the information on individual CI couplings, one has to invert the system of equations (17). The solution reads:

$$
\begin{aligned}
\mathrm{d} \sigma_{\mathrm{RR}} & =\frac{\left(1+P_{2}\right)^{2}}{2 P_{2}\left(P_{1}+P_{2}\right)} \mathrm{d} \sigma_{++}+\frac{\left(1-P_{1}\right)^{2}}{2 P_{1}\left(P_{1}+P_{2}\right)} \mathrm{d} \sigma_{--}-\frac{1-P_{1} P_{2}}{2 P_{1} P_{2}} \mathrm{~d} \sigma_{+-} \\
\mathrm{d} \sigma_{\mathrm{LL}} & =\frac{\left(1-P_{2}\right)^{2}}{2 P_{2}\left(P_{1}+P_{2}\right)} \mathrm{d} \sigma_{++}+\frac{\left(1+P_{1}\right)^{2}}{2 P_{1}\left(P_{1}+P_{2}\right)} \mathrm{d} \sigma_{--}-\frac{1-P_{1} P_{2}}{2 P_{1} P_{2}} \mathrm{~d} \sigma_{+-}, \\
\mathrm{d} \sigma_{\mathrm{LR}} & =-\frac{1-P_{2}^{2}}{2 P_{2}\left(P_{1}+P_{2}\right)} \mathrm{d} \sigma_{++}-\frac{1-P_{1}^{2}}{2 P_{1}\left(P_{1}+P_{2}\right)} \mathrm{d} \sigma_{--}+\frac{1+P_{1} P_{2}}{2 P_{1} P_{2}} \mathrm{~d} \sigma_{+-} .
\end{aligned}
$$


As one can see from Eqs. (15) and (16), contrary to the case of Bhabha scattering, each of the cross sections $\sigma_{\mathrm{RR}}, \sigma_{\mathrm{LL}}$ and $\sigma_{\mathrm{LR}}$ depend on an individual contact interaction parameter, so that full disentanglement of the various couplings (hence the derivation of model-independent constraints) is directly obtained by electron beams polarization in the Møller process.

Similar to Sect. 2.1, see Eqs. (11) and (12), we define measurable event rates integrated over each bin in $z=\cos \theta$ :

$$
N_{++}, \quad N_{--}, \quad N_{+-},
$$

and $(\alpha \beta=++$, etc.):

$$
N_{\alpha \beta}^{\text {bin }}=\frac{1}{3} \mathcal{L}_{\text {int }}\left(e^{-} e^{-}\right) \epsilon \int_{\text {bin }}\left(\mathrm{d} \sigma_{\alpha \beta} / \mathrm{d} z\right) \mathrm{d} z
$$

In Eq. (20), $\mathcal{L}_{\text {int }}$ is the time-integrated luminosity in the $e^{-} e^{-}$mode of the Linear Collider, and is assumed to be equally divided among the three combinations of electron beams polarizations defined in (17). To account for the lower luminosity in the $e^{-} e^{-}$mode due to anti-pinching in the interaction region [5, 21], we assume $\mathcal{L}_{\text {int }}\left(e^{-} e^{-}\right) \simeq \frac{1}{3} \mathcal{L}_{\text {int }}\left(e^{+} e^{-}\right)$. Also, as regards the longitudinal polarization of electrons, we take the symmetric configuration $\left|P_{1}^{-}\right|=\left|P_{2}^{-}\right|=0.8$.

Fig. 4 is the analogue of Fig. 1 for Bhabha scattering and represents the bin-integrated

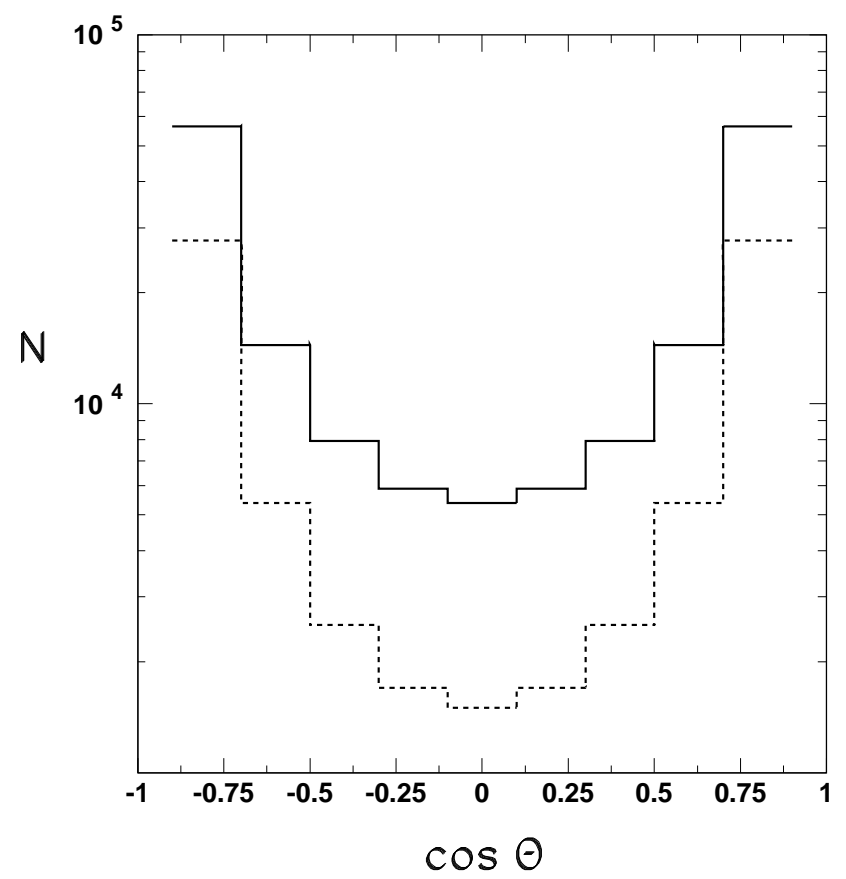

Figure 4: Bin-integrated angular distributions of $N_{++}^{\text {bin }}$ (solid line) and $N_{+-}^{\text {bin }}$ (dashed line) in the $\mathrm{SM}$ at $\sqrt{s}=500 \mathrm{GeV}, \mathcal{L}_{\text {int }}\left(e^{-} e^{-}\right)=\mathcal{L}_{\text {int }}\left(e^{+} e^{-}\right) / 3$ with $\mathcal{L}_{\text {int }}\left(e^{+} e^{-}\right)=50 \mathrm{fb}^{-1}$ and $\left|P_{1}^{-}\right|=\left|P_{2}^{-}\right|=0.8$. 
angular distributions of $N_{++}^{\text {bin }}$ and $N_{+-}^{\text {bin }}$ in the SM, calculated by means of the effective Born approximation, for the c.m. energy and integrated $e^{-}-e^{-}$luminosity indicated in the caption. One should notice, in this case, the peaks in the forward and backward directions, dominated by the $t$ and $u$ photon poles leading to high statistics in those kinematical regions, and the dip at $90^{\circ}$. The $\cos \theta$ distribution for $N_{--}^{\text {bin }}$ has similar features. Relative
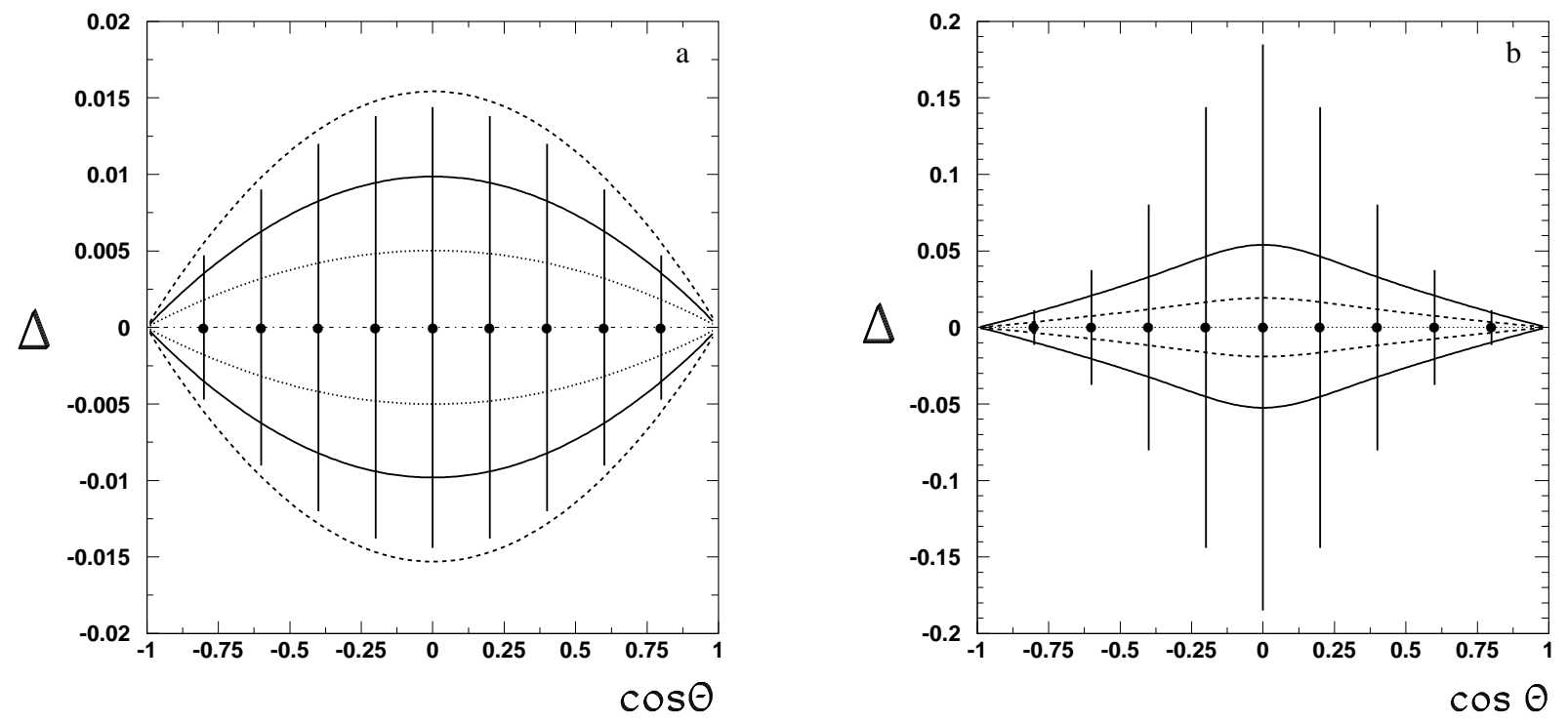

Figure 5: The angular distributions of relative deviations from SM predictions: (a) $\Delta\left(\sigma_{\mathrm{RR}}\right)$ for $\Lambda_{\mathrm{RR}}=40 \mathrm{TeV}$ (dashed line), $50 \mathrm{TeV}$ (solid line) and $70 \mathrm{TeV}$ (dotted line); (b) $\Delta\left(\sigma_{\mathrm{LR}}\right)$ for $\Lambda_{\mathrm{LR}}=30 \mathrm{TeV}$ (solid line) and $50 \mathrm{TeV}$ (dashed line). The curves above (below) the horizontal line correspond to negative (positive) interference between contact interaction and SM amplitude. The error bars show the expected statistical relevant uncertainty at $\mathcal{L}_{\text {int }}\left(e^{-} e^{-}\right)$same as in Fig. 4.

deviations of $\sigma_{\mathrm{RR}}, \sigma_{\mathrm{LL}}$ and $\sigma_{\mathrm{LR}}$ from the SM model due to the contact interactions can be defined in analogy to Eq. (13). In Fig. 5 we show the angular distribution of the deviations $\Delta\left(\sigma_{\mathrm{RR}}\right)$ and $\Delta\left(\sigma_{\mathrm{LR}}\right)$, for the values of $\mathcal{L}_{\text {int }}\left(e^{-} e^{-}\right)$and $\Lambda_{i j}$ indicated in the caption, and with the SM predictions evaluated in the same effective Born approximation used in Fig. 4. Such deviations are compared to the expected statistical uncertainties represented by the vertical bars. The indication of Fig. 5, the analogue of Fig. 2 for Bhabha scattering, is that, in Møller scattering, the sensitivity of $\sigma_{\mathrm{RR}}$ to the related contact parameter $\epsilon_{\mathrm{RR}}$ is almost flat in $\cos \theta$ leading to high sensitivity to $\epsilon_{\mathrm{RR}}$ (the same occurs for $\sigma_{\mathrm{LL}}$ and $\epsilon_{\mathrm{LL}}$ ). Conversely, maximal sensitivity to $\epsilon_{\mathrm{LR}}$ is obtained in the forward and backward regions where the expected statistical uncertainties become smaller. The corresponding behaviour of the statistical significance, defined as the ratio between deviations and uncertainties for each bin, are shown in Fig. 6, the analogue of Fig. 3.

We now proceed to the estimate of the constraints on the contact interaction couplings from the two processes. 

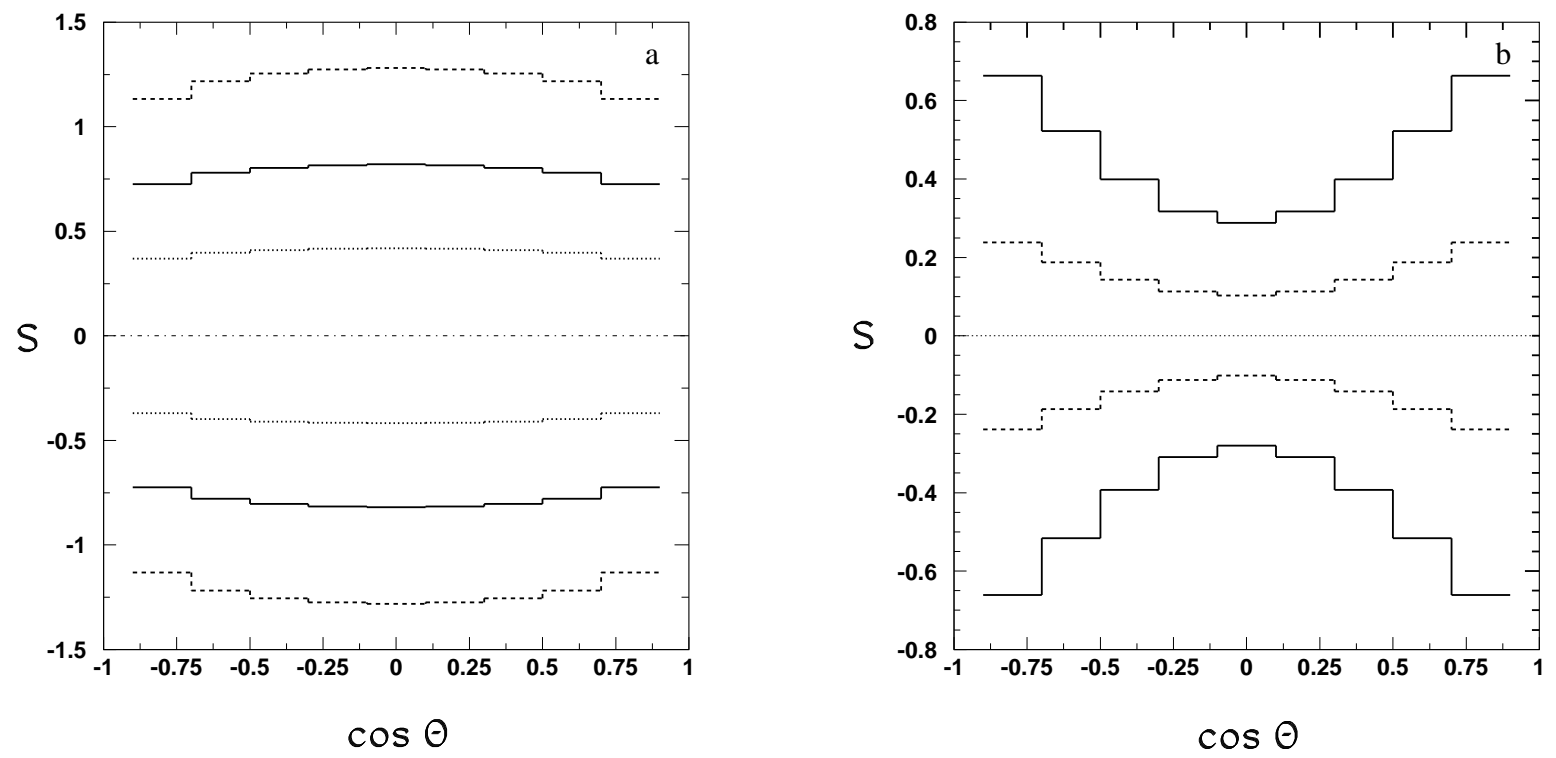

Figure 6: (a) Statistical significance $\mathcal{S}\left(\sigma_{\mathrm{RR}}\right)$ as a function of $\cos \theta$ at $\Lambda_{\mathrm{RR}}=40 \mathrm{TeV}$ (dashed line), $50 \mathrm{TeV}$ (solid line) and $70 \mathrm{TeV}$ (dotted line); (b) Statistical significance $\mathcal{S}\left(\sigma_{\mathrm{LR}}\right)$ as a function of $\cos \theta$ at $\Lambda_{\mathrm{LR}}=30 \mathrm{TeV}$ (solid line) and $50 \mathrm{TeV}$ (dashed line). Here, all inputs are the same as in Fig. 4.

\section{$3 \quad$ Numerical analysis and constraints on CI couplings}

We start by assessing the sensivity of Bhabha scattering to the compositeness scale. To this purpose, we assume the data to be well-described by the SM predictions $\left(\epsilon_{i j}=0\right)$, i.e., that no deviation is observed within the foreseen experimental accuracy, and perform a $\chi^{2}$ analysis of the $\cos \theta$ angular distribution. For each of the observable cross sections, the $\chi^{2}$ distribution is defined as the sum over the above mentioned nine equal-size $\cos \theta$ bins introduced in Sect. 2:

$$
\chi^{2}(\mathcal{O})=\sum_{\text {bins }}\left(\frac{\Delta(\mathcal{O})^{\text {bin }}}{\delta \mathcal{O}^{\text {bin }}}\right)^{2}=\sum_{\text {bins }}\left[\mathcal{S}(\mathcal{O})^{\text {bin }}\right]^{2},
$$

where $\mathcal{O}=\sigma_{\mathrm{L}}, \sigma_{\mathrm{R}}, \sigma_{\mathrm{LR}, t}$ and $\sigma^{\text {bin }} \equiv \int_{\text {bin }}(\mathrm{d} \sigma / \mathrm{d} z) \mathrm{d} z$. In Eq. (21),$\Delta(\mathcal{O})$ represents the relative deviation from the SM prediction defined in Eq. (13), and $\delta \mathcal{O}$ is the expected experimental relative uncertainty, that combines the statistical and the systematic one.

In order to achieve comparable accuracy in experimental measurements and theoretical predictions, radiative corrections to Bhabha and Møller scatterings have to be taken into account [22]. In practice, initial state radiation is by far the most relevant part of the QED modifications 23]. The method that we shall follow to evaluate the effects of the QED radiation for large-angle Bhabha scattering is the one that uses the so called structure function approach [23, 24] where soft and hard photon emission is taken into account. As to Møller scattering, the QED corrections to polarized cross section will be evaluated by 
means of the FORTRAN code MOLLERAD [25, 26], adapted to the present discussion, with $m_{\text {top }}=175 \mathrm{GeV}$ and $m_{H}=120 \mathrm{GeV}$.

Concerning the numerical inputs and assumptions used in the estimate of $\delta \mathcal{O}$, to assess the role of statistics we vary $\mathcal{L}_{\text {int }}\left(e^{+} e^{-}\right)$from 50 to $500 \mathrm{fb}^{-1}$ (a third of the total running time for each polarization configuration of Eq. (9)). As for the systematic uncertainty, we take $\delta \mathcal{L}_{\text {int }} / \mathcal{L}_{\text {int }}=0.5 \%, \delta \epsilon / \epsilon=0.5 \%$ and, regarding the electron and positron degrees of polarization, $\delta P_{1} / P_{1}=\delta P_{2} / P_{2}=0.5 \%$.

As a criterion to constrain the values of the contact interaction parameters allowed by the non-observation of the corresponding deviations, we impose $\chi^{2}<\chi_{\mathrm{CL}}^{2}$, where the actual value of $\chi_{\mathrm{CL}}^{2}$ specifies the desired 'confidence' level. We take the values $\chi_{\mathrm{CL}}^{2}=3.84$ and 5.99 for $95 \%$ C.L. for a one- and a two-parameter fit, respectively.

We begin the presentation of the numerical results from the consideration of $\sigma_{\mathrm{L}}$ and $\sigma_{R}$. Since these cross sections simultaneously depend on the pairs of independent CI couplings $\left(\epsilon_{\mathrm{LL}}, \epsilon_{\mathrm{LR}}\right)$ and $\left(\epsilon_{\mathrm{RR}}, \epsilon_{\mathrm{LR}}\right)$ a two-parameter analysis is needed in these cases. The $95 \% \mathrm{CL}$ allowed areas are represented by the elliptical contours around $\epsilon_{\mathrm{LL}}=\epsilon_{\mathrm{RR}}=\epsilon_{\mathrm{LR}}=0$, depicted in Figs. 7a,b. The maximum reachable values of $\Lambda_{\mathrm{RR}}$ and $\Lambda_{\mathrm{LL}}$ correspond to the minimum of the lower branches of the curves in these figures.
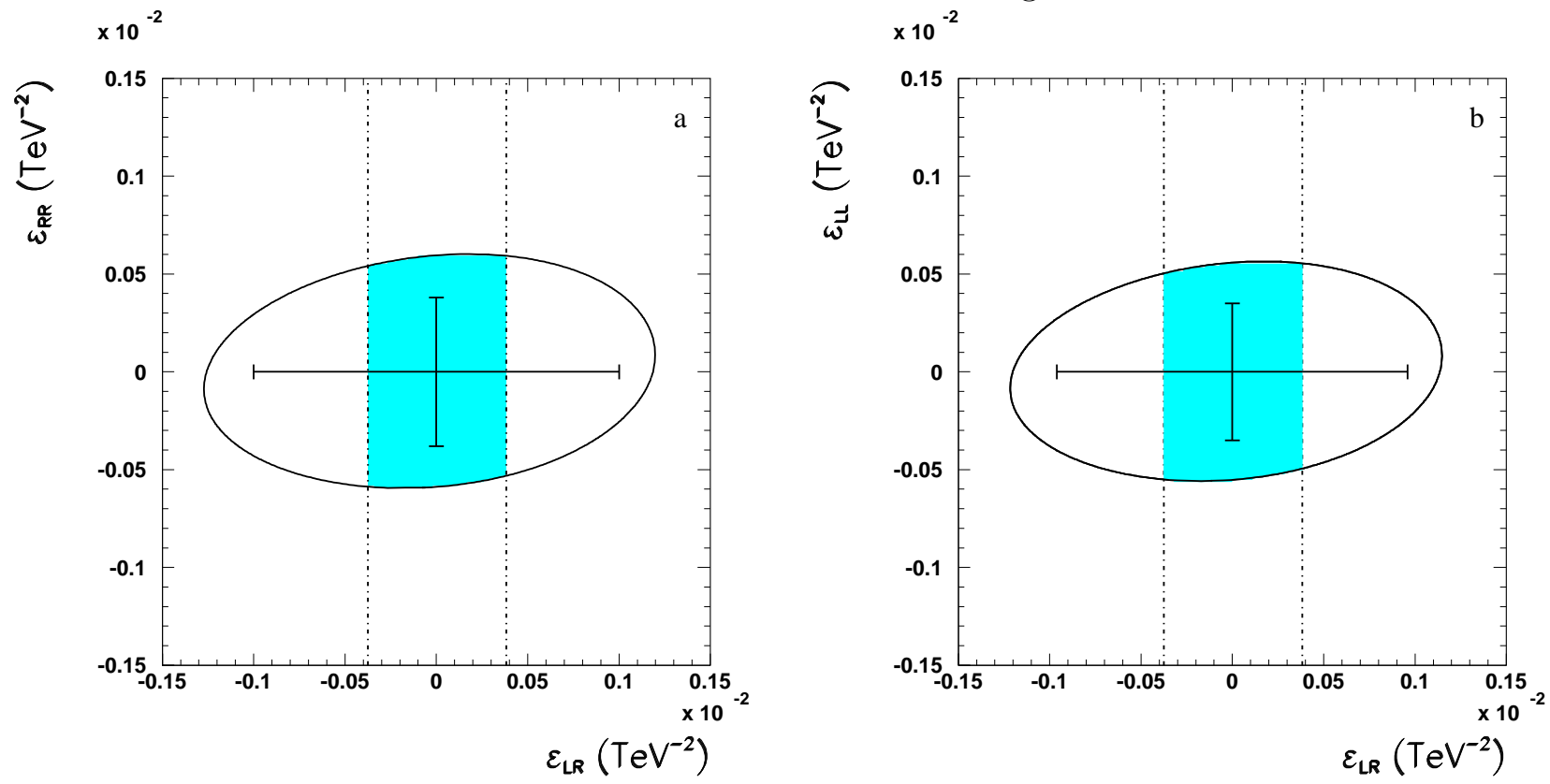

Figure 7: Allowed areas (ellipses) at 95\% C.L. on electron contact interaction parameters in the planes $\left(\epsilon_{\mathrm{LR}}, \epsilon_{\mathrm{RR}}\right)$ and $\left(\epsilon_{\mathrm{LR}}, \epsilon_{\mathrm{LL}}\right)$, obtained from $\sigma_{\mathrm{R}}(\mathrm{a})$ and $\sigma_{\mathrm{L}}(\mathrm{b})$, respectively, at $\sqrt{s}=500 \mathrm{GeV}, \mathcal{L}_{\text {int }}\left(e^{+} e^{-}\right)=50 \mathrm{fb}^{-1},\left|P^{-}\right|=0.8$ and $\left|P^{+}\right|=0.6$. Vertical dashed curves indicate the allowed range for $\epsilon_{\mathrm{LR}}$ obtained from $\sigma_{\mathrm{LR}, t}$.

Turning to $\epsilon_{\mathrm{LR}}$, the relevant cross section $\sigma_{\mathrm{LR}, t}$ depends only on that parameter, see Eqs. (77) and (8), so that the corresponding constraints are determined from a one-parameter fit (with the lower value of $\chi_{\mathrm{CL}}^{2}$ ). The model-independent, discovery reach expected at the 
Linear Collider for the corresponding mass scale $\Lambda_{\mathrm{LR}}$ is represented, as a function of the integrated luminosity $\mathcal{L}_{\text {int }}$, in Fig. 8. As expected, the highest luminosity determines the strongest constraints on the CI couplings 5

The $95 \%$ CL bounds on $\epsilon_{\mathrm{LR}}$ can be reported in Figs. 7a,b to narrow the constraints on $\epsilon_{\mathrm{RR}}$ and $\epsilon_{\mathrm{LL}}$, respectively. They are represented by the vertical lines there, so that the final allowed regions, at the $95 \%$ CL, are the shaded ones. Fig. 8 dramatically shows the really high sensitivity of $\sigma_{\mathrm{LR}, t}$, such that the discovery limits on $\Lambda_{\mathrm{LR}}$ are the highest, compared to the $\Lambda_{\mathrm{RR}}$ and $\Lambda_{\mathrm{LL}}$ case.

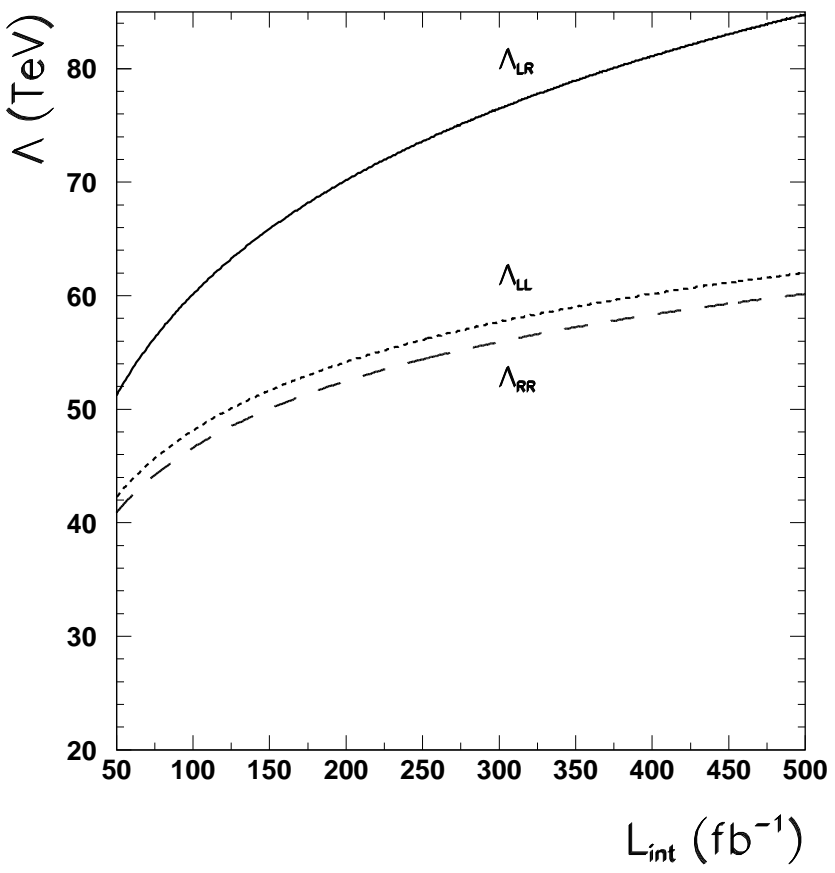

Figure 8: Reach in $\Lambda$ at $95 \%$ C.L. vs. integrated luminosity $\mathcal{L}_{\text {int }}\left(e^{+} e^{-}\right)$obtained from the model-independent analysis for $e^{+}+e^{-} \rightarrow e^{+}+e^{-}$at $E_{\text {c.m. }}=0.5 \mathrm{TeV},\left|P^{-}\right|=0.8$ and $\left|P^{+}\right|=0.6, \Lambda_{\mathrm{LR}}$ (solid line), $\Lambda_{\mathrm{RR}}$ (dashed line), $\Lambda_{\mathrm{LL}}$ (dotted line).

The crosses in Fig. 7a,b represent the model-dependent constraints obtainable by taking only one non-zero parameter at a time, instead of two simultaneously non-zero and independent as in the analysis presented above. The arms of the crosses refer to integrated luminosity $\mathcal{L}_{\text {int }}=50 \mathrm{fb}^{-1}$. One can note from Figs. 7a,b that the 'single-parameter' constraints on the individual CI parameters $\epsilon_{\mathrm{RR}}$ and $\epsilon_{\mathrm{LL}}$ are numerically more stringent, as compared to the model-independent ones. Essentially, this is a reflection of the smaller critical value of $\chi^{2}, \chi_{\text {crit }}^{2}=3.84$, corresponding to $95 \%$ C.L. with a one-parameter fit.

\footnotetext{
${ }^{5}$ Such increase with luminosity is somewhat slower than expected from the scaling law $\Lambda \sim\left(s \mathcal{L}_{\text {int }}\right)^{1 / 4}$ [27, since with our input choice the effect of the systematic uncertainties can compete with the statistical one.
} 
The procedure, and the criteria, to derive numerical constraints from the Møller process are quite similar, the outstanding difference being that, in this case, each measurable cross section in (18) depends on a single contact interaction parameter, so that complete disentangling of $\epsilon$ 's is directly obtained and the smaller $\chi_{\mathrm{CL}}^{2}=3.84$, relevant to one-parameter cases, applies. Certainly, this is an advantage if one wants to perform a model-independent analysis of electron contact interactions. Also, substantially higher longitudinal polarization should be attainable for electron beams than for positron ones, for a given luminosity. On the other side, there is the penalty of the lower luminosity expected in the $e^{-} e^{-}$mode, depressing the sensitivity, and, as previously stated, in our examples we have assumed a third of the luminosity in the $e^{+} e^{-}$mode. The lower bounds on $\Lambda$ 's, derived under these conditions, are shown as a function of the integrated luminosity in Fig. 9.

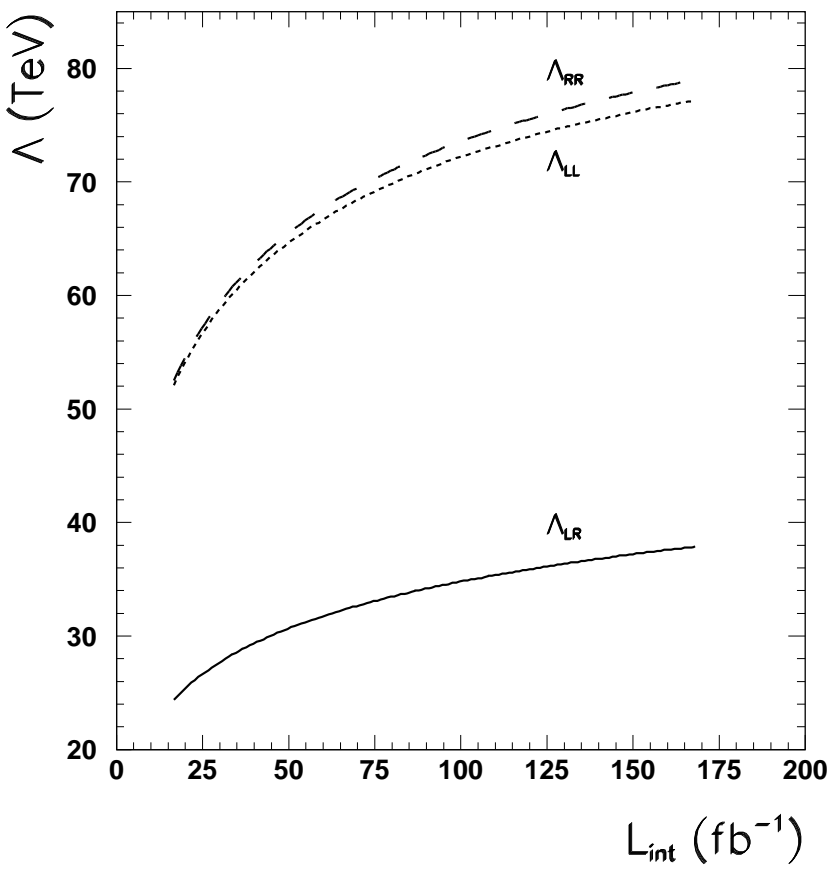

Figure 9: Reach in $\Lambda$ at $95 \%$ C.L. vs. integrated luminosity $\mathcal{L}_{\text {int }}\left(e^{-} e^{-}\right)$obtained from the model-independent analysis for $e^{-}+e^{-} \rightarrow e^{-}+e^{-}$at $E_{\mathrm{c} . \mathrm{m} .}=0.5 \mathrm{TeV},\left|P_{1}^{-}\right|=\left|P_{2}^{-}\right|=0.8$, $\Lambda_{\mathrm{LR}}$ (solid line), $\Lambda_{\mathrm{RR}}$ (dashed line), $\Lambda_{\mathrm{LL}}$ (dotted line).

\section{Concluding remarks}

In the previous sections we have derived limits on the electron contact interactions by simultaneously considering Bhabha scattering and Møller scattering at a Linear Collider with longitudinally polarized beams, using a model-independent analysis that allows to 
simultaneously account for all independent couplings as non-vanishing free parameters. The analysis is based on the definition of measurable polarized differential cross sections that allows to derive: $i$ ) from Bhabha scattering, separate bounds on $\epsilon_{\mathrm{LR}}$ and in the planes $\left(\epsilon_{\mathrm{LL}}, \epsilon_{\mathrm{LR}}\right)$ and $\left(\epsilon_{\mathrm{RR}}, \epsilon_{\mathrm{LR}}\right)$; ii) from Møller scattering, completely individual bounds on $\epsilon_{\mathrm{LL}}$, $\epsilon_{\mathrm{RR}}$ and $\epsilon_{\mathrm{LR}}$. Numerical results for the lower bounds on the corresponding range in the relevant mass scales $\Lambda_{i j}$, depending on the luminosity, are shown in Figs. 8 and 9 , and are summarized in Table 1. Essentially, for c.m. energy $\sqrt{s}=500 \mathrm{GeV}$ and reasonable assumptions on luminosities, polarizations and their relative uncertainties, in the Bhabha mode the bounds vary from 41 to $62 \mathrm{TeV}$ for the LL and RR cases, and from 51 to $85 \mathrm{TeV}$ for the LR coupling. In the Møller mode, the bounds vary from 52 to $79 \mathrm{TeV}$ for the LL and RR cases, and from 24 to 38 for the LR coupling (notice the reduced luminosity input in this case). Therefore, one can conclude that the two processes are complementary as far as the sensitivity to the individual couplings in a model-independent data analysis is concerned: the sensivity of Bhabha scattering to $\Lambda_{\mathrm{LR}}$ is dramatically higher, while Møller scattering is the most sensitive to $\Lambda_{\mathrm{LL}}$ and $\Lambda_{\mathrm{RR}}$. Basically, for the inputs used in Figs. 8 and 9 , the ratio of the maximal sensitivities to $\Lambda$ of the two processes has the qualitative behaviour:

$$
\frac{\Lambda_{\mathrm{RR}}^{e^{-} e^{-}}}{\Lambda_{\mathrm{LR}}^{e^{+} e^{-}}} \approx\left(2 \frac{\mathcal{L}_{\mathrm{int}}\left(e^{-} e^{-}\right)}{\mathcal{L}_{\mathrm{int}}\left(e^{+} e^{-}\right)}\right)^{1 / 4} \approx 0.9
$$

Table 1: Reach in $\Lambda_{i j}$ at 95\% C.L., from the model-independent analysis performed for $e^{+} e^{-} \rightarrow \mu^{+} \mu^{-}$and $e^{+} e^{-}$, at $E_{\text {c.m. }}=0.5 \mathrm{TeV}, \mathcal{L}_{\text {int }}=50 \mathrm{fb}^{-1}$ and $500 \mathrm{fb}^{-1},\left|P^{-}\right|=0.8$ and $\left|P^{+}\right|=0.6$.

\begin{tabular}{|c|c|c|c|c|c|}
\hline process & $\begin{array}{c}\mathcal{L}_{\text {int }} \\
\mathrm{fb}^{-1}\end{array}$ & $\begin{array}{c}\Lambda_{\mathrm{LL}} \\
\mathrm{TeV}\end{array}$ & $\begin{array}{c}\Lambda_{\mathrm{RR}} \\
\mathrm{TeV}\end{array}$ & $\begin{array}{c}\Lambda_{\mathrm{LR}} \\
\mathrm{TeV}\end{array}$ & $\begin{array}{c}\Lambda_{\mathrm{RL}} \\
\mathrm{TeV}\end{array}$ \\
\hline \multirow{2}{*}{$e^{+} e^{-} \rightarrow \mu^{+} \mu^{-}$} & 50 & 35 & 35 & 31 & 31 \\
\cline { 2 - 6 } & 500 & 47 & 49 & 51 & 52 \\
\hline \multirow{2}{*}{$e^{+} e^{-} \rightarrow e^{+} e^{-}$} & 50 & 42 & 41 & 51 & \\
\cline { 2 - 7 } & 500 & 62 & 60 & 85 & \\
\cline { 2 - 7 }$e^{-} e^{-} \rightarrow e^{-} e^{-}$ & $50 / 3$ & 52 & 53 & 24 & \\
\cline { 2 - 7 } & $500 / 3$ & 77 & 79 & 38 & \\
\hline
\end{tabular}

All this shows the benefits of initial beams longitudinal polarization, that allows, by measuring suitable combinations of polarized cross sections, to directly disentangle the individual couplings. Indeed, as previously observed, in general without polarization only correlations among contact interaction parameters, rather than finite allowed regions, could be derived and consequently, in the unpolarized case, only a one-parameter analysis, relating to a specific model, can be performed.

In Table 1 we have also reported the numerical results relevant to the annihilation into muon pairs, derived from a similar analysis [10]. 
As an example of application of the obtained results to a possible source of contact interactions, we may consider the sneutrino parameters (mass $m_{\tilde{\nu}}$ and Yukawa coupling

$\lambda$ ) envisaged by supersymmetric theories with $\mathcal{R}$-parity breaking. In this case, sneutrino exchange affects only those helicity amplitudes with non-diagonal chiral indices, so that $\Lambda_{\mathrm{LR}}$ is the relevant mass scale [28, 29]. Qualitatively, without entering into a detailed and more complex analysis, one can expect typical bounds on $m_{\tilde{\nu}} / \lambda \sim \Lambda_{\mathrm{LR}} / \sqrt{8 \pi} \simeq 10$ to 17 $\mathrm{TeV}$ corresponding to $\Lambda_{\mathrm{LR}} \approx 51 \mathrm{TeV}$ and $85 \mathrm{TeV}\left(\right.$ Fig. 8) at $\mathcal{L}_{\text {int }}=50 \mathrm{fb}^{-1}$ and $500 \mathrm{fb}^{-1}$, respectively.

\section{Acknowledgements}

This research has been partially supported by MIUR (Italian Ministry of University and Research) and by funds of the University of Trieste.

\section{References}

[1] See, e.g. G. Pasztor and M. Perelstein, SNOWMASS-2001-P315, Nov. 2001, hep-ph/0111471;

S. Cullen, M. Perelstein and M. Peskin, Phys. Rev. D 62 (2000) 055012.

[2] T. G. Rizzo, SLAC-PUB-9295, hep-ph/0208027.

[3] E. Eichten, K. Lane and M. E. Peskin, Phys. Rev. Lett. 50 (1983) 811;

R. Rückl, Phys. Lett. B 129 (1983) 363.

[4] J. A. Aguilar-Saavedra et al., DESY-2001-011, hep-ph/0106315.

T. Abe et al, SLAC-R-570, hep-ex/0106055.

[5] Proceedings of the Electron-Electron Linear Collider Workshop, Dec. 1999, Santa Cruz (CA), Ed. C. A. Heusch, Int. J. Mod. Phys. A15, No. 16, 2000.

[6] C. Geweniger et al., Combination of the LEP II $f \bar{f}$ Results, LEP2FF/01-02 (2001).

[7] D. Bourilkov, Phys. Rev.D 64 (2001) 071701.

[8] K. Cheung, Phys. Lett. B 517 (2001) 167;

V. Barger and K. Cheung, Phys. Lett. B 480 (2000) 149; and references there.

[9] A. F. Zarnecki, Nucl. Phys. Proc. Suppl. 79 (1999) 158.

[10] A. A. Babich, P. Osland, A. A. Pankov and N. Paver, LC Note LC-TH-2001-021 (2001), hep-ph/0101150; Phys. Lett. B 518 (2001) 128.

[11] B. Schrempp, F. Schrempp, N. Wermes and D. Zeppenfeld, Nucl. Phys. B 296 (1988) 1 .

[12] D. Bardin, W. Hollik and T. Riemann, Z. Phys. C 49 (1991) 485. 
[13] E. J. Eichten and S. Keller, in Physics at the First Muon Collider, Nov. 1977, Batavia, IL, hep-ph/9801258.

[14] M. Beccaria, F. M. Renard, S. Spagnolo and C. Verzegnassi, Phys. Rev. D 62 (2000) 053003.

[15] For earlier discussions of polarization in processes (11) and (2) see, e.g.:

R. Gastmans and Y. Van Ham, Phys. Rev. D 10 (1974) 3629;

L. L. Deraad, Phys. Rev. D 11 (1975) 3328;

M. J. Puhala, T. G. Rizzo and B. L. Young, Phys. Lett. B 109 (1982) 411;

H. A. Olsen and P. Osland, Phys. Rev. D 25 (1982) 2895;

S. Jadach and B. F. L. Ward, Phys. Rev. D 54 (1996) 743, and references there.

[16] M. Consoli, W. Hollik and F. Jegerlehner, CERN-TH-5527-89, presented at the Workshop on $Z$ Physics at LEP.

[17] G. Altarelli, R. Casalbuoni, D. Dominici, F. Feruglio and R. Gatto, Nucl. Phys. B 342 (1990) 15.

[18] F. Cuypers, PSI-PR-96-32, hep-ph/9611336, published in New directions in highenergy physics, Eds. D. G. Cassel, L. Trindle Gennari and R. H. Siemann, p. 878.

[19] F. Cuypers and P. Gambino, Phys. Lett. B 388 (1996) 211.

[20] A. Czarnecki and W. J. Marciano, Int. J. Mod. Phys. A 15 2365; Phys. Rev. D 53 (1996) 1066.

[21] J. E. Spencer, Int. J. Mod. Phys. A 11 (1996) 1675.

[22] A. Denner and S. Pozzorini, Eur. Phys. J. C 7 (1999) 185;

M. Bohm, A. Denner and W. Hollik, Nucl. Phys. B 304 (1988) 687.

[23] G. Montagna, O. Nicrosini and, in Radiative Corrections for $e^{+} e^{-}$Collisions, Ed. J. H. Kühn (Springer, Berlin, 1989), p.25;

O. Nicrosini and L. Trentadue, in QED Structure Functions, Ed. G. Bonvicini, AIP Conf. Proc. No. 201 (AIP, New York, 1990), p.12.

[24] G. Montagna, O. Nicrosini and F. Piccinini, Phys. Rev. D 48 (1993) 1021.

[25] V. A. Mosolov, N. M. Shumeiko and J. G. Suarez, see ref. [5], Int. J. Mod. Phys. A 15 (2000) 2377.

[26] N. M. Shumeiko and J. G. Suarez, J. Phys. G 26 (2000) 113.

[27] T. L. Barklow, Int. J. Mod. Phys. A 11 (1996) 1579.

[28] T. G. Rizzo, Phys. Rev. D 59 (1999) 113004. 
[29] J. Kalinowski, R. Rückl, H. Spiesberger and P. M. Zerwas, Phys. Lett. B 406 (1997) 314. 